\title{
STUDY ON COLD/WARM SENSATION OF MATERIALS USED IN DESKTOP OF FURNITURE
}

\author{
Wen-Gang Hu, Na Liu, Lu Xu, Huiyuan Guan \\ Nanjing Forestry University \\ CHINA
}

(Received August 20I9)

\begin{abstract}
The aim of this study was to investigate the cold/warm sensations of materials used in desktop when forearms touching desktop. Both experimental tests and subjective evaluations were conducted in this study. A device was developed used to simulate forearm in order to replace subjects. Five men and five women were selected and introduced to six types of materials and two types of environmental temperatures in the tests. The results showed that the effects of environmental temperature on contact temperature of all tested materials were statistically significant, and the differences among wood and wood based materials, plastic materials, and artificial stone were also statistically significant. The device developed in this study was qualified to measure the contacting temperature between forearm and desktop sustainably and steadily, which can reduce the error introduced by subjects. Although qualitative relation was found between contact temperature and subjective evaluations, no quantitative correlation was proved.
\end{abstract}

KEYWORDS: Desktop, furniture, forearm, cold/warm sensation.

\section{INTRODUCTION}

Man has a very effective temperature regulatory system, which keeps the body's core temperature at approximately $37^{\circ} \mathrm{C}$. Sweating is an effective cooling tool because the energy required for the sweat to evaporate is taken from the skin. Man considers the environment comfortable if no type of thermal discomfort is present. The first comfort condition is thermal neutrality, which means that a person feels neither too warm nor too cold. Franger (1972) regarded thermal neutrality as thermal comfort. Gagge and Gonzalez (1974) thought thermal comfort is a comfortable condition that you do not feel cold or hot. It was defined in the ISO 7730 (1994) standard as being "That condition of mind which expresses satisfaction with the thermal environment". A definition most people can agree on, but also a definition which is not easily converted into physical parameters. 
Thermal environments are considered together with other factors such as air quality, light, noise level and interior decoration, when we evaluate our working environment (Song et al. 2016). If we do not feel the everyday working environment is satisfactory, our working performance will inevitably suffer. Thus, thermal comfort also has an impact on our work efficiency. Furniture is the most frequent device we touch in daily life and working and occupies most spaces of room. Therefore, it directly influences the thermal environment and comfort.

Some studied the effects of furniture arrangement on the whole thermal environment of room by computational fluid dynamics (Zukowska et al. 2012, Zhuang et al. 2014, Horikiri et al. 2015, Li et al. 2020). Others investigated the thermal comfort of human body when touching furniture (Zhou et al. 2018). Wang et al. (2007) studied how upper extremity skin temperatures correlate with overall-body thermal sensation by measuring skin temperatures at the finger, hand, and forearm. The results showed that finger temperature and finger-forearm temperature gradients are very similar in their correlation to overall sensation. Wang et al. (2000) investigated the relation between the skin temperature of the palm and sensory cold-warmth after contact with some materials. Results showed that it is possible to evaluate the contacted sensory cold-warmth relying on the basic thermal properties of material. In addition, gender difference in thermal comfort for Chinese people was investigated through two laboratory experiments by Lan et al. (2007). The results show that there are gender differences in thermal comfort for Chinese people. Female comfortable operative temperature $\left(26.3^{\circ} \mathrm{C}\right)$ is higher than male comfortable operative temperature $\left(25.3^{\circ} \mathrm{C}\right)$. Pasut et al. (2013) evaluated the effect of heated/cooled chair on thermal sensation and comfort. The results show that the heated/cooled chair strongly influences the subjects' thermal sensation and comfort. Vlavić et al. (2012a) studied temperature and moisture as the contributing factors to sitting comfort during performance of usual tasks under controlled conditions. The study method employed temperature and moisture measurements on five different office chairs using the probes placed on or in the seats. At the same year, they further studied the thermal discomfort of five different office chairs as subjects performed their usual jobs in controlled conditions by sensation differential technique (Vlaović et al. 2012b).

Forearm is the part touching the desktop most frequently, and the skin is most sensitive to temperature when contacting with desktop, therefore, sensations of forearm for temperature contacting with desktop plays an important role in thermal comfort of man.

The aim of this study was to evaluate the cold/warm sensation of materials commonly used in desktop when forearm contacts with desktops using experimental test and subjective evaluation. The specific objectives are to 1) evaluate the changes of temperature when forearm contacting different desktop materials by subjects; 2) develop a simple device to simulate the forearm used to measure the changes of temperature when forearm contacting desktop; 3) evaluate the cold/warm by seven-point thermal sensation scale semantics differential method; 4) compare the results of experimental tests measured by subjects, device and subjective evaluation.

\section{MATERIAL AND METHODS}

\section{Test materials}

Six types of materials commonly used in desktop (Chen and Zhu 2019, Guo et al. 2020) were chosen in this study including medium density fiberboard (MDF), laminated bamboo lumber (LBL) rubber wood (RW), melamine resin surfaced particleboard (MRSP), acrylonitrile butadiene styrene plastic (ABS), and marble (MARB). The dimensions of specimens are all measured $300 \mathrm{~mm}$ long, $150 \mathrm{~mm}$ wide and $18 \mathrm{~mm}$ thick. All samples were conditioned in a humidity chamber controlled at $20^{\circ} \mathrm{C} \pm 2{ }^{\circ} \mathrm{C}$ and $50 \% \pm 3 \%$ relative humidity (RH) for two weeks. 


\section{Equipment and device}

Thermocouple thermometers (Xinsite, HT-9815, China) was used to measure the changes of temperature, which contains four channels, and the measuring range is from $50^{\circ} \mathrm{C}$ to $300^{\circ} \mathrm{C}$ with accuracy of $0.1^{\circ} \mathrm{C}$.

Fig. 1 shows the device developed in this study to simulate forearms of subjects with outline dimensions of $250 \mathrm{~mm} \times 100 \mathrm{~mm} \times 70 \mathrm{~mm}$. It is composed of seven parts: (1) digital display temperature controller used to control temperature, (2) power supply, (3) temperature probe used to measure internal temperature of the device, (4) heating plate, (5) elastic silicone rubber sheet used to simulate skin of man, (6) Aluminum alloy U-groove is supporting structure, and (7) thermal insulation cotton used to keep internal temperature of device.

The work principle of device is to simulate the temperature of forearm by internal temperature of the device, and the surface of elastic silicone rubber sheet is used to simulate skin of forearm.

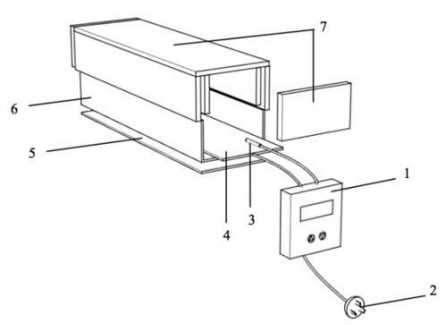

(a)

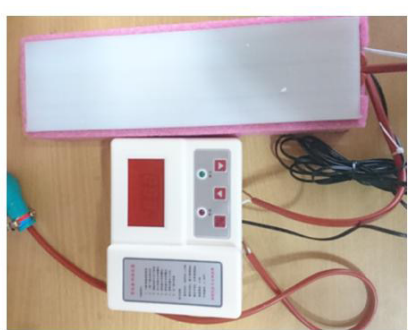

(b)

Fig. 1: The device used to simulate forearm (a) diagram and (b) the physical photo.

\section{Test methods}

The thermal comfort is seriously influenced by environmental temperature. Therefore, two environmental temperatures were set to investigate the sensations of cold/warm for different desktop materials, the high temperature (HT) and low temperature (LT) are $25^{\circ} \mathrm{C}$ and $18^{\circ} \mathrm{C}$, respectively. The relative humidity keeps at a constant $50 \% \pm 3 \%$. The experiment includes two parts to conduct, the first one is to measure the temperature changes of forearm by subjects and device developed in the study respectively, and the second one is subjective evaluation on cold/ warm sensation by subjects with semantics differential (SD) method (Zhagn et al. 2020).

\section{Test measured by subjects}

Ten healthy subjects with five males and five females were selected in this study. All of them are between 23-25 years old staffs. In order to minimize the effect of clothing, the subjects all wore the same cotton T-shirts, and jeans. Measurements were carried out in the air-conditioned room. Prior to measurement, the environmental temperature was turned to the specified temperature (HT or LT), and subjects went into the laboratory $30 \mathrm{~min}$ before conducting test to make the body temperature adjust to the environment.

Fig. 2 presents the setup of test. The tested specimen was laid on two foam strips to prevent from conducting heat through lab table, and the thermocouple probe was attached to the center of the forearm of subject with breathable tape. The temperature of subjects' forearm skins were measured before started testing. Subjects put their forearm right upon the tested specimen and kept in naturally reading postures. Each test lasted for $30 \mathrm{~min}$, the temperature changes were record by the thermocouple thermometer with $1 \mathrm{~min}$ interval within the first $6 \mathrm{~min}$ of test, and recorded with increment of 3 min after 6 min. Each subject took 10 min break after finishing 
each type of material test, each subject was tested in high and low temperature environment, respectively, in morning and afternoon. All tests were conducted throughout 10 days.

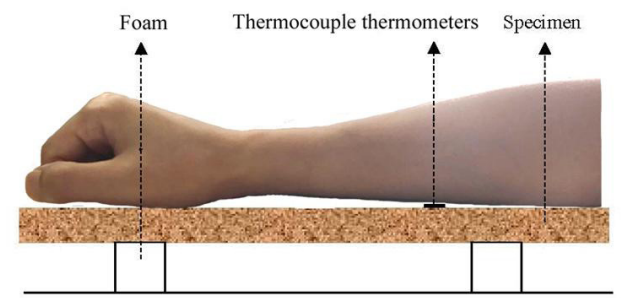

Fig. 2: Setup for measuring the temperature between desktop and forearm.

\section{Test measured by device}

Forearm of subject was replaced by the device developed in this study and the same test was repeated using the above testing method. Prior to measurement, the temperature of elastic silicone rubber sheet must be close to that of forearm skin as much as possible. In order to achieve this goal, correction must be done to make sure the correlation between internal temperature of device and the temperature of elastic silicone rubber sheet. The internal temperature of device was set as $T_{O}$ with values $35^{\circ} \mathrm{C}, 36^{\circ} \mathrm{C}, 37^{\circ} \mathrm{C}, 38^{\circ} \mathrm{C}$, and $39^{\circ} \mathrm{C}$ respectively, and then the temperature of elastic silicone rubber sheet was set as $T$, and the values of $T$ corresponding to each $T_{0}$ can were measured by thermocouple thermometer. Finally, the correlation between $T_{0}$ and $T$ was figured out by regression method.

\section{Subjective evaluation}

During the experimental test by subjects, the subjects were also asked to evaluate the thermal comfort of tested materials by SD method after starting the test $1 \mathrm{~min}, 5 \mathrm{~min}, 15 \mathrm{~min}$, and $30 \mathrm{~min}$.

Tab. 1: Seven-point thermal sensation scale.

\begin{tabular}{|c|c|c|c|c|c|c|c|}
\hline Semantic & Cold & Cool & Slightly cool & Neutral & Slightly warm & Warm & Hot \\
\hline Scale & -3 & -2 & -1 & 0 & 1 & 2 & 3 \\
\hline
\end{tabular}

Tab. 1 is the 7-point thermal sensation scale used to evaluate the thermal sensation according to Franger (1972) and ISO 7730 (1994). Fanger found that being thermally neutral guarantees comfort, because in optimal condition, no specific sensation of feeling warm or cold is to be expected by the subjects (Candas 2005).

\section{RESULTS AND DISCUSSION}

\section{Results of experiments measured by subjects}

Fig. 3 shows the temperature changes of six types of materials in HT and LT environments. The temperature-time curves all present the same trends, the temperature increased proportionally with time dramatically within the first $1 \mathrm{~min}$, and then the curve increased slowly and temperature tended to be a constant at the end of test. In addition, the temperature of MARB was lower than other materials no matter in HT or LT environment, especially in LT environment. 


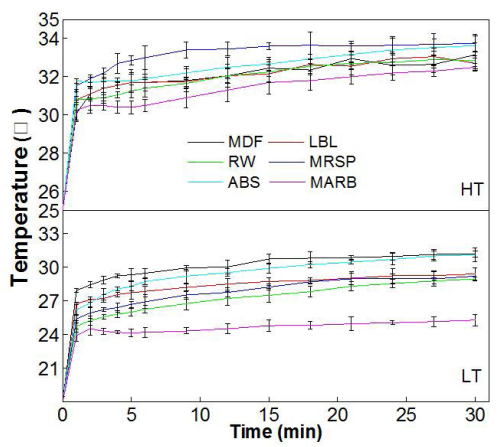

Fig. 3: Results of experiments measured by subjects.

An analysis of variation (ANOVA) was conducted to analyze the effects of material type and environmental temperature on contacting temperature of forearm at the time point of $30 \mathrm{~min}$. The results showed the effects of environmental temperature on contacting temperature of forearm with all tested materials were statistically significant, and the differences between wood/wood based materials (MDF, WR, and LBL), plastic materials (ABS, and MESP), and artificial stone (MARB) were also statistically significant with their p-values all below 0.05 .

\section{Results of experiments measured by device}

The correlation between $T$ and $T_{0}$ was regressed by Origin pro 9.1 software (OriginLab, 1991), the regression equations in high and low temperature environments are shown in Eq. 1 and Eq. 2 respectively.

$$
\begin{array}{ll}
\mathrm{T}_{\mathrm{HT}}=0.1171 \mathrm{~T}_{0}^{2}-7.8246 \mathrm{~T}_{0}+161.79 & \mathrm{R}^{2}=0.99988 \\
\mathrm{~T}_{\mathrm{LT}}=0.0857 \mathrm{~T}_{0}^{2}-5.7569 \mathrm{~T}_{0}+125.5 & \mathrm{R}^{2}=0.99553
\end{array}
$$

where: $\quad T_{H T} / T_{L T}$-temperature of elastic silicone rubber sheet in high/low environment, $\left({ }^{\circ} \mathrm{C}\right)$, $T_{O}$ - is internal temperature of the device, $\left({ }^{\circ} \mathrm{C}\right)$.

The temperature-time curves of all six types of materials measured by device are all in the same trends compared with those determined with subjects, which is shown in Fig. 4. In addition, the temperature of MARB was still the lowest of all materials, which was consistent with results measured by subjects. 


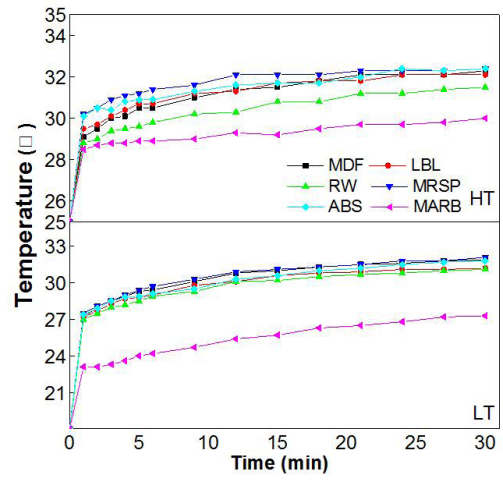

Fig. 4: Results of experiments measured by device.

\section{Comparison of experimental results measured by subjects and device}

The comparisons of results measured by subjects and device in HT and LT environments are shown in Fig. 5 and Fig. 6, resp.

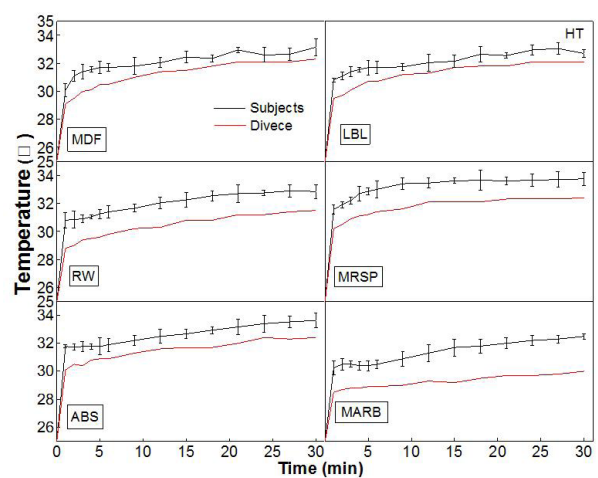

Fig. 5: Comparison of the results measured by subjects and device in high temperature environment.

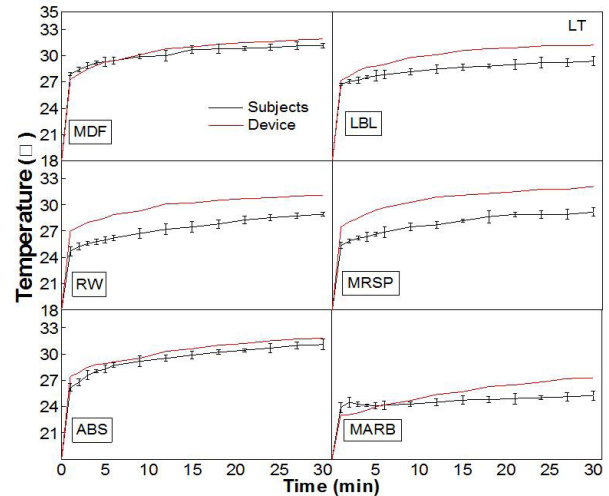

Fig. 6: Comparison of the results measured by subjects and device in low temperature environment.

The trends of temperature-time curves of them were similar to each other. However, the temperatures measured by subjects were all bigger than those measured by device in HT environment. By contrast, the results measured by device were bigger than those determined by subjects in LT environment. Furthermore, the temperatures of every measured time point of subject and device were compared in HT and LT environments, and the average errors and coefficients of variation (COV) are shown in Tab. 2. It suggested that the errors between the results of subjects and those of device were all within 10\%, which means that device developed in this study can be used to measure the temperature between forearm and desktop sustainably and steadily. 
Tab. 2: Errors of device measurement in high and low temperature environments.

\begin{tabular}{|c|c|c|}
\hline Error & HT & LT \\
\hline MDF & $3.04 \%(38.4)$ & $1.51 \%(56.8)$ \\
\hline GLB & $2.89 \%(33.4)$ & $5.09 \%(32.8)$ \\
\hline RW & $5.04 \%(12.7)$ & $9.21 \%(10.0)$ \\
\hline MFCB & $4.43 \%(10.4)$ & $9.72 \%(8.8)$ \\
\hline ABS & $3.46 \%(20.4)$ & $2.68 \%(35.1)$ \\
\hline MARBLE & $6.48 \%(16.5)$ & $4.35 \%(60.6)$ \\
\hline
\end{tabular}

\section{Results of subjective evaluation}

Fig. 7 and Fig. 8 are results of mean values of subjective evaluations scored by subjects at time point of $1 \mathrm{~min}, 5 \mathrm{~min}, 15 \mathrm{~min}$ and $30 \mathrm{~min}$ after starting tests in HT and LT environments respectively. According to the definition of thermal comfort (Franger 1972), subjects feel thermal comfort, when condition is not too hot or cold. In other words, the subjects are in thermal neutrality when sensation scale is equal to 0 . Thus, the order of thermal comfort for all materials is: LBL, MDF, WR, MARB, MRSP, and ABS successfully in HT environment. The order in LT environment is: WR, MDF, MRSP, LBL, ABS, and MARB. Although the gender of subject is different, there is no gender difference in thermal sensation near neutral conditions (Lan et al. 2007).

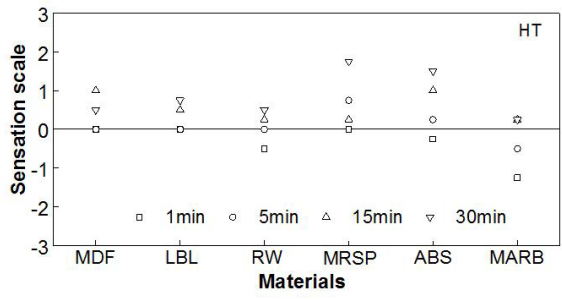

Fig. 7: Results of subjective evaluations of subjects in high temperature environment.

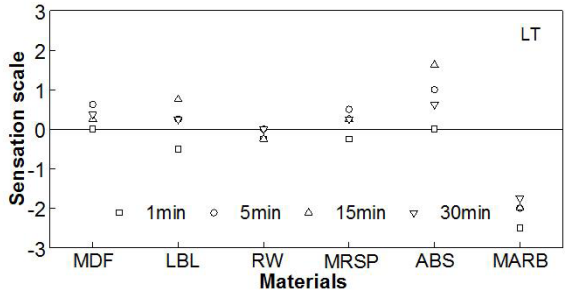

Fig. 8: Results of subjective evaluations of subjects in low temperature environment.

To be specific, the RW and MDF performed better thermal comfort than other tested materials both in HT and LT environments. The reason is that the thermal conductivities of wood and wood based materials are smaller than those of the other tested materials used in this study, because of the porosity of timber. The thermal comfort of MARB in HT environment was better than LT environment. The thermal conductivity of MARB is bigger than those of the other tested materials used in the study (Wang et al. 2001, Yang et al. 2016, Wang et al. 2020, Tang and Fu 2020), which made it adjusted to the environment temperature soon. Therefore, in HT environment, subjects felt cool at first, and then felt neutral with the temperature increasing. By contrast, in LT environment, the subjects felt more and more cold sensation with the temperature decreasing. In addition, in HT environment, the average sensation scale of ABS was -0.25 at $1 \mathrm{~min}$, which means subjects felt slightly cool. However, with time increasing, the sensation scale increased fast to 1.5 at the end of test, which means the subject felt warm. It suggests that the sensation of cold/warm of material is not absolute but relative, which seriously influenced by environmental temperature and contacting time (Gagge et al. 1967, Höppe 2002). 
To some extent, it can be found that there was a qualitative relationship between contact temperature and sensation of cold/warm. However, no correlation was found between the temperature and subjective evaluations of subjects, which was also confirmed by Vlaović et al. (2012b).

\section{CONCLUSIONS}

The cold/warm sensations of forearm when contacting different desk top materials in HT and LT environments were studied by experimental and subjective methods. Following conclusions were drawn: (1) The effects of environmental temperature on contacting temperature of forearm contacting all desktop materials were statistically significant. In specific, the differences between wood and wood based materials (MDF, WR, and LBL), plastic materials (ABS, and MESP), and artificial stone (MARB) were all statistically significant respectively. (2) The device developed in this study was qualified to measure the contacting temperature between forearm and desktop sustainably and steadily, which can be used to replace the subjects, and reduce the error introduced by subjects. (3) Although some qualitative relation was found between contacting temperature and subjective evaluations, no quantitative correlation was proved between them.

The thermal comfort is so complex and important for man that further studies are worth being done. The influences of different veneer materials on thermal comfort of desktop and how to choose materials of desktop for different types of furniture are two interesting topics to further study.

\section{ACKNOWLEDGMENTS}

This work was supported by a Scientific Research Foundation of Metasequoia teacher (163104060). A Project Funded by the Nation First-class Disciplines (PNFD), and A Priority Academic Program Development of Jiangsu Higher Education Institutions (PAPD).

\section{REFERENCES}

1. Candas, V., 2005: To be or not to be comfortable: Basis and prediction. Environmental ergonomics: The ergonomics of human comfort, health and performance in the thermal environment. In: Tochihara, Y., Ohnaka, T. (Eds.), Elsevier ergonomics book series, Vol. 3, Elsevier Ltd., Pp 207-215.

2. Chen Y.S., Zhu J., 2019: Study on bending characteristics of fast growing eucalyptus bookcase shelves by using burgers model. Wood Research 64(1): 137-144.

3. Fanger, P.O., 1972: Thermal comfort. McGraw Hill. New York, 25 pp.

4. Gagge, A.P., Gonzalez, R.R., 1974: Physiological and physical factors associated with warm discomfort in sedentary man. Environmental Research 7(2): 230-242.

5. Gagge, A.P., Stolwijk, J.A.J., Hardy, J.D., 1967: Comfort and thermal sensations and associated physiological responses at various ambient temperatures. Environmental Research 1 (1): 1-20.

6. Guo, Y., Guo, C.X., Shi, X., Shen, L.M., 2020: Adaptability of study desks and chairs based on analysis of sitting posture using OpenPose. Journal of Forestry Engineering 5(2): 179-185. 
7. Höppe, P., 2002: Different aspects of assessing indoor and outdoor thermal comfort. Energy and Buildings 34(6): 661-665.

8. Horikiri, K., Yao, Y.F., Yao, J., 2015: Numerical optimization of thermal comfort improvement for indoor environment with occupants and furniture. Energy and Buildings 88: 303-315.

9. ISO 7730, 1994: Moderate thermal environments. Determination of the PMV and PPD indices and specification of the conditions for thermal comfort.

10. Lan, L., Lian, Z.W., Liu, W.W., Liu, Y.M., 2007: Investigation of gender difference in thermal comfort for Chinese people. European Journal of Applied Physiology 102(4): 471-480.

11. Li, X.X., Shen, L.M., Gao, J.S., 2020: Investigation of influence mechanism of temperature-controlled mattress on human thermal comfort in winter. Journal of Forestry Engineering 5(3): 175-182.

12. Pasut, W., Zhang, H., Kaam, S., Arens, E., Zhai, Y., 2013: Effect of a heated and cooled office chair on thermal comfort. HVAC\&R Research 19(5): 574-583.

13. Song, S.S., Wan, Q. Wang, G.G., 2016: Eye movement evaluation of different wood interior decoration space. Wood Research 61(5): 831-843.

14. Tang, T.L., Fu, Y.C., 2020: Formation of chitosan/sodium phytate/nano- $\mathrm{Fe}_{3} \mathrm{O}_{4}$ magnetic coatings on wood surfaces via layer-by-layer self-assembly. Coatings 10(1): 51.

15. Vlaović, Z., Domljan, D., Grbac, I., 2012a: Research of temperature and moisture during sitting on office chairs. Drvna Industrija 63(2):105-112.

16. Vlaović, Z., Domljan, D., Župčić, I., Grbac, I., 2012b: Thermal comfort while sitting on office chairs-subjective evaluations. Drvna Industrija 63 (4): 263-270.

17. Wang, D., Zhang, H., Arens, E., Huizenga, C., 2007: Observations of upper-extremity skin temperature and corresponding overall-body thermal sensations and comfort. Building and Environmental 42(12): 3933-3943.

18. Wang, S.Y., Lin, F.C., Lin, M.Y., 2000: Thermal properties of interior decorative material and contacted sensory cold-warmth I: Relation between skin temperature and contacted sensory cold-warmth. Journal of Wood Science 46: 357-363.

19. Wang, S.Y., Lin, F.C., Lin, M.Y., 2001: Thermal properties of interior decorating material and the sensation of cold/warm by contact II: the relations among heat flux, temperature change of material, and sensation of cold/warm by contact. Journal of Wood Science 47: 109-114.

20. Wang, W.L., Peng, J.D., Zhao, Z.Y., Zhou, X.J., Zhang, J., Du, G.B., 2020: Modification of melamine-formaldehyde resin for decoration board. Journal of Forestry Engineering 5(2): 42-47.

21. Yang, B.S., He, S.J., Wang, F., Que, Z.L., Pan, B., Zhu, Y.X., 2016: Thermal performance of electrically heated flooring prepared by thin Chinese fir glulam. Journal of Forestry Engineering 1(1): 46-50.

22. Zhagn, Z.H., Xu, B.M., 2020: Tang dynasty chair feature design based on kansei evaluation and eye tracking system. Wood Research 65(1): 161-174.

23. Zhou, C.M., Yu, M.N., Zhou, T., 2018: Experimental study on three-dimensional shape mapping of complex furniture. EURASIP Journal on Image and Video Processing 9: 89.

24. Zhou, Z.B., Li, X., Ding, J.W., Guo, X.L. Cao, P.X., 2019: Structure design and process analysis of internal heating engineered wood flooring. Journal of Forestry Engineering 4(1): 165-169. 
25. Zhuang, R., Li, X., Tu, J., 2014: CFD study of the effects of furniture layout on indoor air quality under typical office ventilation schemes. Building Simulation 7: 263-275.

26. Zukowska, D., Melikov, A.K., Popiolek, Z., 2012: Impact of personal factors and furniture arrangement on the thermal plume above a sitting occupant. Building and Environmental 49: 104-116.

\author{
Wen-Gang Hu, Na Liu, Lu Xu, Huiyuan Guan* \\ Nanjing Forestry University \\ Co-Innovation Center of Efficient Processing and Utilization of Forest \\ Resources \\ College of Furnishings and Industrial Design \\ NANJING \\ CHINA \\ *Corresponding author: hwg@njfu.edu.cn
}

